\title{
Review
}

\section{Transport of silicon from roots to panicles in plants}

\author{
By Jian Feng MA, ${ }^{* 1, \dagger}$ Naoki YAmaJI*1 and Namiki MitanI-UENO*1 \\ (Communicated by Teruhiko BePPU, M.J.A.)
}

\begin{abstract}
Silicon ( $\mathrm{Si}$ ) is the most abundant minerals in soil and exerts beneficial effects on plant growth by alleviating various stresses. The transport of Si from soil to the panicles is mediated by different transporters. Lsi1, belonging to a NIP group of the aquaporin family, is responsible for the uptake of $\mathrm{Si}$ from soil into the root cells in both dicots and monocots although its expression patterns and cellular localization differ with plant species. The subsequent transport of $\mathrm{Si}$ out of the root cells towards the stele is medicated by an active efflux transporter, Lsi2. Lsi1 and Lsi2 are polarly localized at the distal and proximal sides, respectively, of both exodermis and endodermis in rice root. Silicon in the xylem sap is presented in the form of monosilicic acid and is unloaded by Lsi6, a homolog of Lsi1 in rice. Lsi6 is also involved in the inter-vascular transfer of Si at the node, which is necessary for preferential Si distribution to the panicles.
\end{abstract}

Keywords: localization, distribution, uptake, silicon, transporter

\section{Introduction}

Silicon $(\mathrm{Si})$ is the second most abundant element after oxygen in soil. Because of its strong affinity with oxygen, in nature $\mathrm{Si}$ always exists as silica or silicate, which are combined with various metals. Silicon dioxide comprises 50 to 70 percent of the soil mass. Most soils have $\mathrm{Si}$ in the solution between 100 to $500 \mu \mathrm{M}$ in the form of monosilicic acid $\left.\left(\mathrm{H}_{4} \mathrm{SiO}_{4}\right){ }^{1}{ }^{1}\right)$ Therefore, all plants grown in soil will contain some $\mathrm{Si}$ in their tissues although the $\mathrm{Si}$ concentration in the shoots varies considerably among plant species, ranging from $0.1 \%$ to $10 \% \mathrm{Si}$ in the dry weight. ${ }^{2)-4)}$

$\mathrm{Si}$ has not been recognized as an essential element for plant growth. The major reason is that there is no evidence to show that $\mathrm{Si}$ is involved in the metabolism of plant, which is one of the three criteria required for essentiality established by Arnon and Stout. ${ }^{5)}$ However, the beneficial effects of Si on the growth have been reported in a wide of plant species, which are characterized by protecting the plant from various biotic and abiotic stresses.

*1 Institute of Plant Science and Resources, Okayama University, Japan.

$\dagger$ Correspondence should be addressed: Jian Feng Ma, Institute of Plant Science and Resources, Okayama University, 220-1 Chuo, Kurashiki 710-0046, Japan (e-mail: maj@rib.okayamau.ac.jp).
Si enhances resistance of plants to diseases caused by both fungi and bacteria in different plant species. For example, in rice, Si reduces the epidemics of both leaf and panicle blast at different growth stages. ${ }^{6)} \mathrm{Si}$ also decreases the incidence of powdery mildew in cucumber, barley and wheat; sheath blight in rice; ring spot in sugarcane; rust in cowpea; leaf spot in bermuda grass and gray leaf spot in St. Augustine grass and perennial ryegrass. ${ }^{7)}$

Silicon also suppresses insect pests such as stem borer, brown planthopper, rice green leafhopper, whitebacked planthopper and noninsect pests such as leaf spider and mites (Fig. 1A). ${ }^{3), 8)}$ Resistance to the damage by wild rabbit in wheat is also enhanced by an increased amount of Si in wheat. ${ }^{9}$ )

Si plays an important role in alleviating various abiotic stresses including physical stress and chemical stress. ${ }^{10)}$ For example, Si can alleviate water stress, climatic stress such as typhoons, low temperature and insufficient sunshine during the summer season. ${ }^{2), 3), 11), 12)}$ Excess transpiration from the husk causes white head, resulting in low fertility in rice (Fig. 1B), however, $\mathrm{Si}$ is effective to protect the panicles from excess transpiration due to heavy deposition of Si on the husk. The beneficial effects of Si under chemical stresses including P-deficiency, $\mathrm{P}$ excess, $\mathrm{Mn}$ and salt toxicity have been observed in many plants. ${ }^{3)}$ Mn toxicity in pumpkin is significantly reduced by $\mathrm{Si}$ application (Fig. 1C). ${ }^{13)}$ 

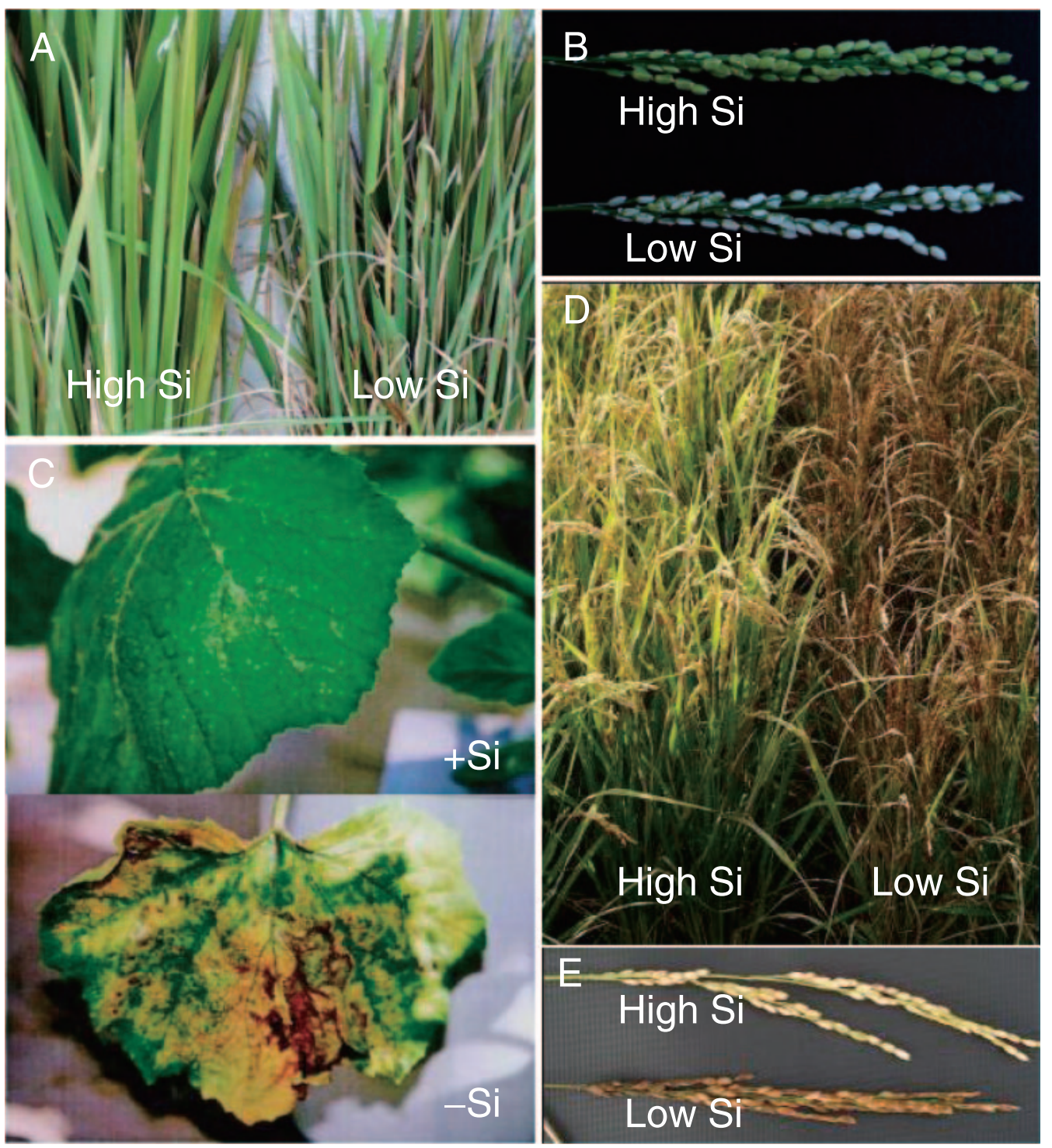

Fig. 1. Examples showing beneficial effects of Si in plants. A, Effect of Si on pest damage. B, Protective role of Si in excess transpiration of panicles in rice. C, Alleviative effect of $\mathrm{Si}$ on $\mathrm{Mn}$ toxicity in pumpkin. The plants were exposed to high $\mathrm{Mn}$ in the presence $(+\mathrm{Si})$ or absence $(-\mathrm{Si}$ ) of $\mathrm{Si}$. D-E, Decreased fertility due to low $\mathrm{Si}$ in rice. A-B, D-E, wild type rice (High $\mathrm{Si}$ ) and a mutant defective in $\mathrm{Si}$ uptake (Low $\mathrm{Si}$ ) were grown in the field. From Tamai and Ma (2008). ${ }^{15)}$

In addition to the role of $\mathrm{Si}$ in alleviating various stresses, Si improves light interception by keeping leaves erect, thereby stimulating canopy photosynthesis in rice. ${ }^{3)}$ This is particularly important in dense plant stands and when nitrogen fertilizers are heavily applied, to minimize mutual shading.

Silicon is especially important for healthy growth and high production of rice, ${ }^{13)-15)}$ which is able to accumulate Si to over $10 \%$ of its dry weight in the shoots. When Si accumulation is not enough, the yield is greatly reduced mainly due to decreased fertility (Fig. 1D, E).${ }^{15)}$ For this reason, Si fertilizers are applied in paddy field in some countries including Japan.

All beneficial effects of Si are mostly attributed to its deposition in different organs. For example, Si is deposited beneath the cuticle to form a cuticle-Si double layer in the leaf (Fig. 2). ${ }^{3), 10)}$ This layer can mechanically impede penetration by fungi and pest and thereby avoiding the infection process. Therefore, to benefit from $\mathrm{Si}$, a high accumulation is required. In this review, we focus on Si transporters involved in the uptake, translocation and distribution, which are identified in our lab. 

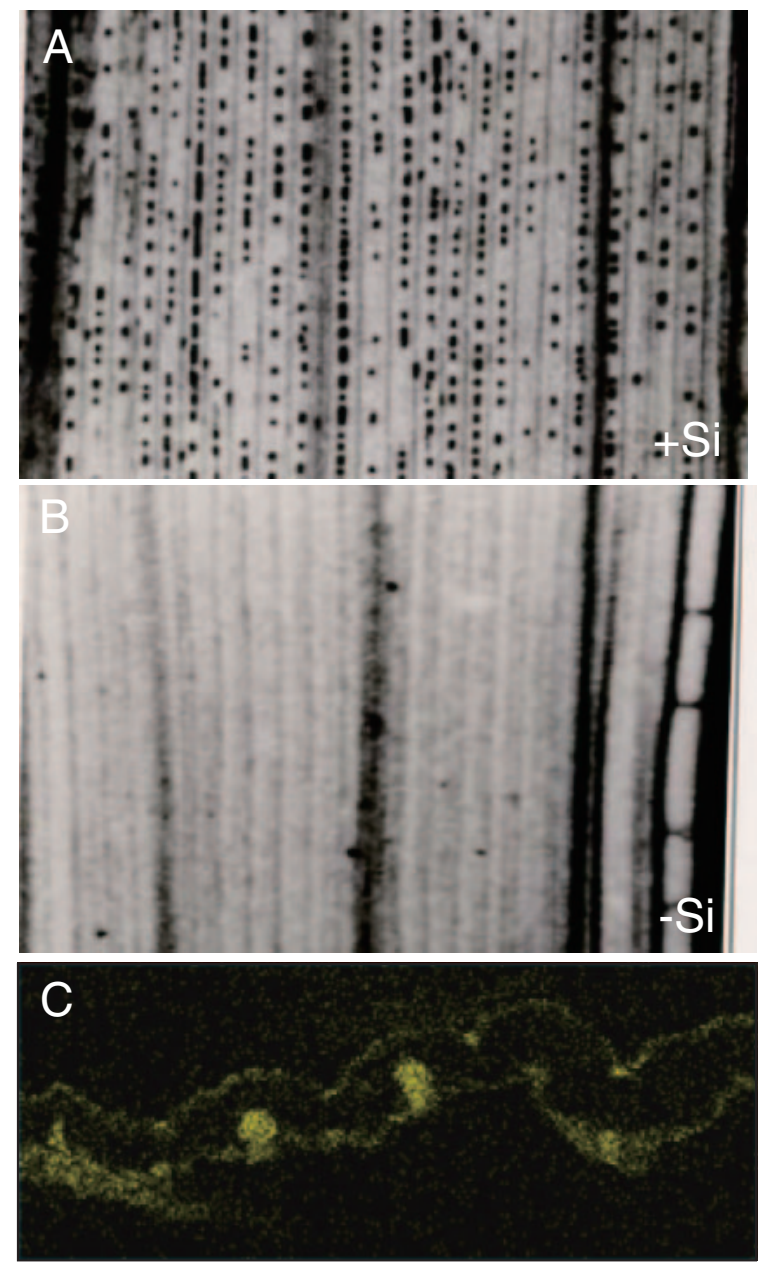

Fig. 2. Deposition of Si in rice leaf blade. A-B, soft X-ray image of rice leave with (A) or without (B) Si. Black dot shows silica bodies. C, SEM-EDX image of a cross section of rice leaf blade. Yellow color shows $\mathrm{Si}$ deposition under cuticle and bulliform motor cells known as plant opal.

\section{Transporters involved in Si uptake}

Plant roots take up Si in the form of silicic acid $\left[\mathrm{Si}(\mathrm{OH})_{4}\right]$. To identify genes that are responsible for Si uptake in rice, we isolated rice mutants with a low Si content. We used tolerance to germanium (Ge) as an indicator for screening rice mutants defective in $\mathrm{Si}$ uptake. Germanium is an analogue of Si and plant roots take up Si and Ge similarly. However, once it is taken up, Ge is toxic to plants, as shown by the characteristic brown spots on the leaf blades (Fig. 3A). ${ }^{16)}$ By using this approach, we have isolated two independent mutants (designated lsi1 and lsi2 for low Si 1 and 2) showing tolerance to Ge from different mutagenized seeds. lsi1 mutant shows similar growth as wild-type rice in the absence of Ge (Fig. 3B). ${ }^{16)}$ However, in the presence of $\mathrm{Ge}$, the growth of lsi1 mutant is hardly inhibited, in contrast to the wild-type rice, whose growth is severely inhibited by Ge (Fig. 3C). The Si uptake of lsi1 is much lower than that of wild-type rice, indicating that this is a mutant defective in $\mathrm{Si}$ uptake. By using lsi1 mutant, the first Si transporter Lsi1 was identified by map-based cloning technique. ${ }^{17)}$ Lsi1 belongs to a Nod26-like major intrinsic protein (NIP) subfamily of aquaporin-like proteins and shows influx activity for silicic acid in Xenopus oocyte. The predicted amino acid sequence has six transmembrane domains and two Asn-Pro-Ala (NPA) motifs, which is well conserved in typical aquaporins. Lsi1 is constitutively expressed in the roots, but its expression is decreased to one fourth by Si supply. ${ }^{17)}$ Recently, it was found that Si accumulated in the shoots but not in the roots regulates the expression of Lsi1. ${ }^{18)}$ Within a root, the expression of Lsi1 is much lower in the root tip region between $0-10 \mathrm{~mm}$ than in the basal regions of the root $(>10 \mathrm{~mm}) .{ }^{19)}$ Silicon uptake in the root tip region ( 0 to $10 \mathrm{~mm}$ ) comprising both the apical meristem and the elongation zone is also much lower than that in the basal regions ( $>10 \mathrm{~mm}$ from the root tips). Therefore, the site of $\mathrm{Si}$ uptake is located in the mature regions of the roots rather than in the root tips. Lsi1 is localized in the main and lateral roots, but not in root hairs. ${ }^{17)}$ In the roots including seminal, lateral and crown roots, the Lsi1 protein is localized to the plasma membrane of both exodermis and endodermis, ${ }^{17)}$ where the Casparian strips prevents apoplastic transport into the root stele. Furthermore, Lsi1 shows polar localization at the distal side of both the exodermis and endodermis cells (Fig. 4A).

Homologs of rice Lsi1 (OsLsi1) have been identified in barley (HvLsi1), maize (ZmLsi1) and pumpkin (CmLsi1). ${ }^{20)-22)}$ However, different from OsLsi1, HvLsi1 from barley and ZmLsi1 from maize are localized at epidermal, hypodermal and cortical cells. ${ }^{20), 21)}$ CmLsi1 from the pumpkin is localized at all root cells. ${ }^{22}$ Interestingly, mutation at the position of 242 from proline to leucine in CmLsi1 resulted in significant decrease in $\mathrm{Si}$ uptake in some pumpkin cultivars due to failure to the localization at the plasma membrane. ${ }^{22)}$ In contrast to OsLsi1, the expression levels of HvLsi1 and ZmLsi1 are unaffected by $\mathrm{Si}^{20), 21)}$

The second $\mathrm{Si}$ transporter Lsi2 in rice was also identified by mutant approach as Lsi1. However, 

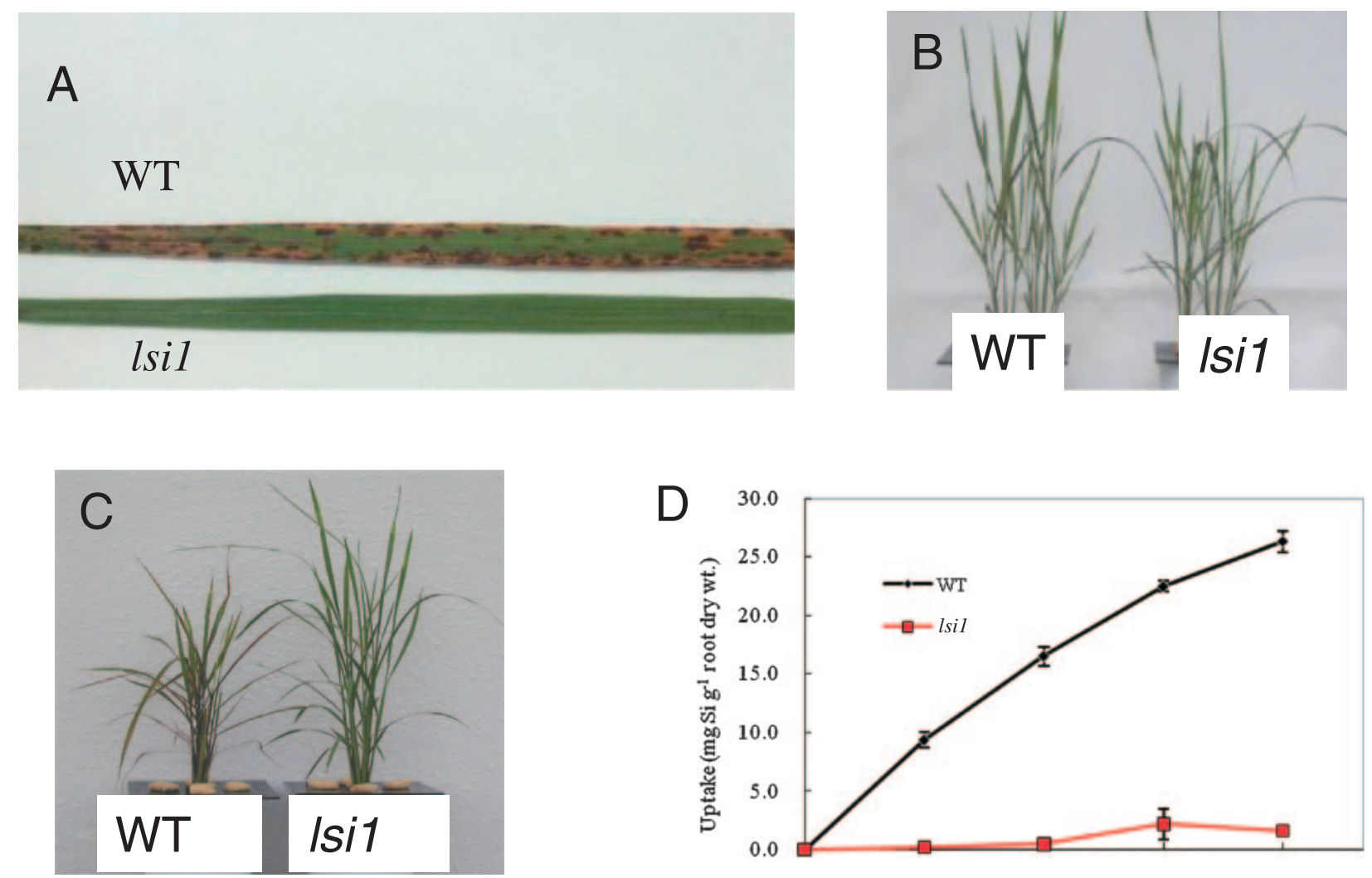

Fig. 3. Phenotype of lsi1 mutant. A, Symptoms of Ge toxicity in the leaf of wild-type rice (WT) and mutant lsi1. B-C, Growth of WT and $l$ si1 in the absence (B) and presence (C) of Ge. D, Si uptake in the mutant and WT. From Ma et al. (2002). ${ }^{16)}$
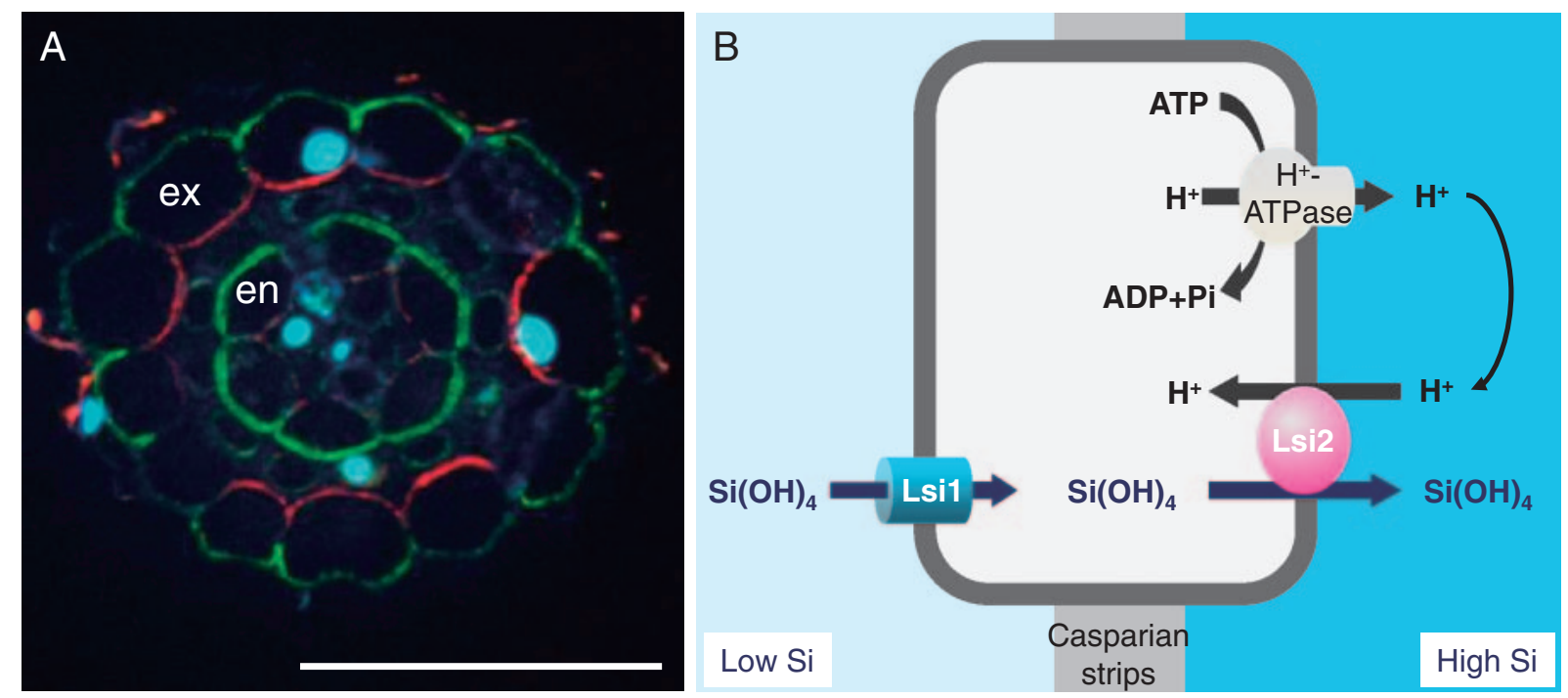

Fig. 4. Localization of Si transporters in rice. A, Polar localization of influx Si transporter Lsi1 (green) and efflux Si transporter Lsi2 (red) in lateral roots. Ex, exodermis; En, endodermis; nuclei stained by DAPI (cyan). From Yamaji and Ma (2011). ${ }^{18)}$ B, Schematic presentation of Si uptake system in rice root exodermis/endodermis cells. 
A Maize/barley

\section{Casparian strip}

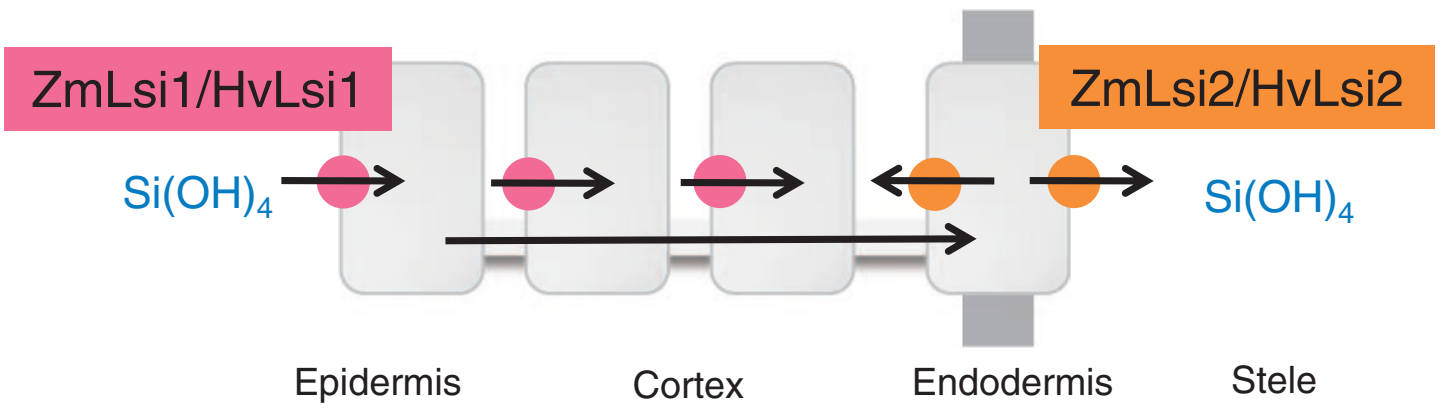

B Rice

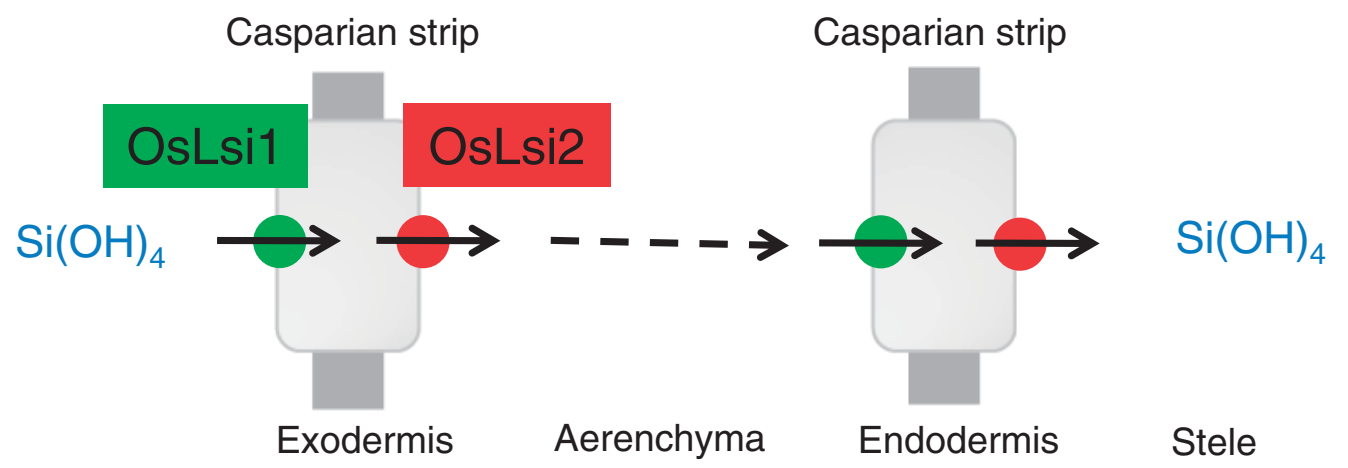

Fig. 5. Schematic presentation of different Si uptake system in barley/maize (A) and rice (B) roots. From Mitani et al. (2009). ${ }^{21)}$

different from Lsi1, Lsi2 is an efflux transporter of Si in rice. ${ }^{23)}$ Lsi2 belongs to a putative anion transporter without any similarity with the silicon influx transporter Lsi1. The expression pattern and tissue- and cellular-localization of Lsi2 is the same as that of Lsi1, ${ }^{23)}$ but in contrast to Lsi1, Lsi2 is localized at the proximal side of the exodermis and the endodermis cells (Fig. 4A). Transport of Si by Lsi2 is driven by the proton gradient (Fig. 4B).

Similar transporters of Lsi2 have also been identified in barley and maize. ${ }^{24}$ However, ZmLsi2 and HvLsi2 are localized only to the endodermis of roots in maize and barley without polarity. These differences result in different pathway of $\mathrm{Si}$ from external solution to the xylem between upland crops (barley and maize) and paddy crop (rice). In barley and maize, Si can be taken up from external solution (soil solution) by HvLsi1/ZmLsi1 at different cells including epidermal, hypodermal and cortical cells (Fig. 5A). In contrast, in rice, $\mathrm{Si}$ is only taken up at the exodermal cells by OsLsi1 (Fig. 5B). After being taken up into the root cells, $\mathrm{Si}$ is transported to the endodermis by symplastic pathway and then released to the stele by HvLsi2/ZmLsi2 in maize and barley (Fig. 5B). By contrast, in rice, Si taken up by OsLsi1 at the exodermal cells is released by OsLsi2 to the apoplast and then transported into the stele by both OsLsi1 and OsLsi2 again at the endodermal cells (Fig. 5B).

The difference in the uptake system may be attributed to the root structures. In rice roots, there are two Casparian strips at the exodermis and endodermis, whereas one Casparian strip is usually present at the endodermis of maize and barley roots under non-stressed conditions. Moreover, mature roots in rice have a distinct structure, a highly developed aerenchyma, wherein almost all cortex cells between exodermis and endodermis are destructed. Therefore, Si transported into the exodermis cells by the influx transporter, OsLsi1, has to be 
released by the efflux transporter, OsLsi2, into the apoplast of a spoke-like structure across the aerenchyma. However, in maize and barley roots, there is no such structure or if any; it is developed poorly. These differences in the localization of transporters and polarity may be one of the reasons for the different $\mathrm{Si}$ uptake capacities among species.

\section{Transporter involved in translocation of $\mathrm{Si}$ from roots to the shoots}

Following uptake by the roots through Lsi1 and Lsi2, Si is translocated to the shoot by transpirational volume flow through the xylem. More than $90 \%$ of Si taken up by the roots is translocated to the shoots in rice. ${ }^{3)}$ The Si concentration in the rice xylem sap could be as high as $20 \mathrm{mM}$. Chemically, silicic acid polymerizes into silica gel $\left(\mathrm{SiO}_{2} \cdot \mathrm{nH}_{2} \mathrm{O}\right)$ when the concentration of silicic acid exceeds $2 \mathrm{mM}$. However, $\mathrm{Si}$ in the xylem sap is presented in the form of monosilicic acid. ${ }^{25), 26)}$ It seems that silicic acid in the xylem sap at high concentration is transiently present because it starts to polymerize in vitro. ${ }^{26}$ The transporter for xylem loading of Si has not been identified.

Silicic acid must be exported from the xylem to other leaf cells. Based on similarity with Lsi1, Lsi6 was isolated and found to be responsible for this process. ${ }^{27)}$ Lsi6 also shows transport activity for silicic acid. However, different from Lsi1 and Lsi2, Lsi6 is also expressed in the leaf sheaths and leaf blades in addition to the root tips. Lsi6 is localized in the adaxial side of the xylem parenchyma cells in the leaf sheaths and leaf blades (Fig. 6A). Knockout of Lsi6 does not affect the uptake of Si by the roots, but affects the silica deposition pattern in the leaf blades and sheaths. ${ }^{27}$ ) There are two types of silicified cells in rice leaf blades; silica cells, and silica bodies or silicified motor cells (Fig. 2B). ${ }^{3), 28)}$ When lsi6 is knocked out, a part of abaxial epidermal cells was also observed to be silicified. Also in the leaf sheaths, the silicified epidermal cells were frequently observed in the knockout line, which are not present in the wild-type rice. These changes in the knockout lines are caused by altered pathway of Si due to loss of function of Lsi6 (Fig. 6B).

Rice is known to produce guttation fluid during the night, a phenomenon of xylem sap exudation through hydathodes, which are located near terminal tracheids of the bundle ends around the margins of leaves due to positive xylem pressure. ${ }^{29)}$ Knockout of lsi6 also causes increased excretion of $\mathrm{Si}$ in the guttation fluid in rice due to altered pathway (Fig. 6B). ${ }^{27)}$
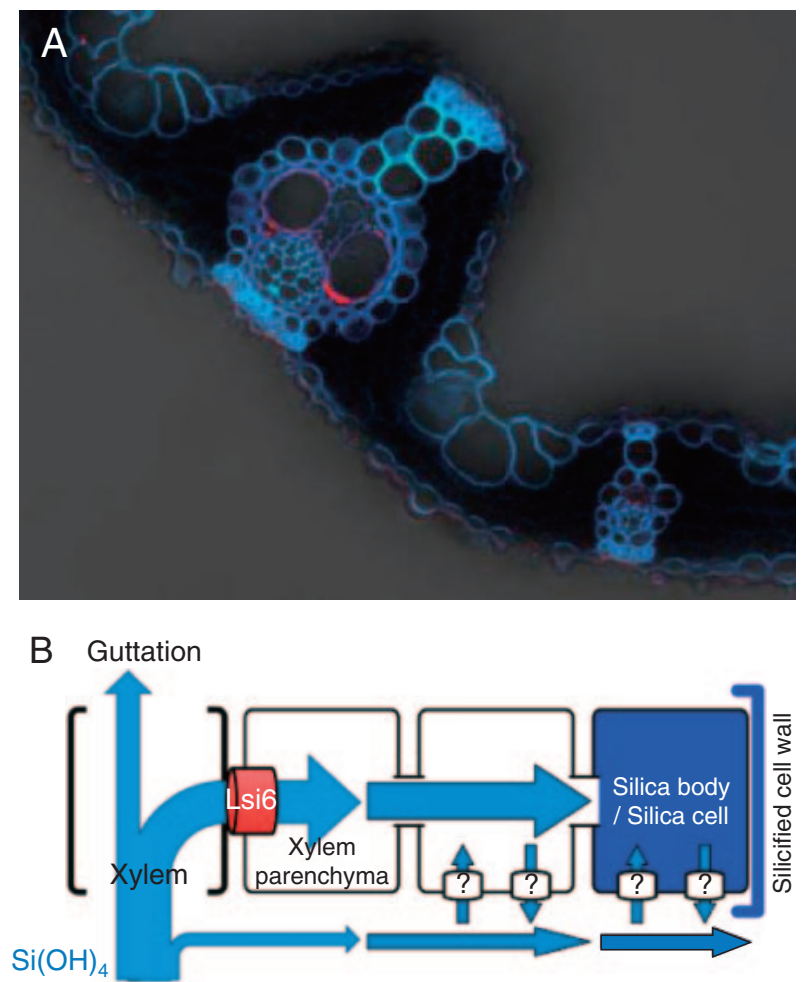

Fig. 6. Localization of Lsi6 in rice leaf blade. A, Immunostaining of Lsi6 (red) showing localization at xylem parenchyma cells. B, Pathway of Si from leaf xylem via Lsi6. From Yamaji et al. $(2008) .^{27)}$

All these observations indicate that Lsi6 is required for delivering $\mathrm{Si}$ to the specific cells. Therefore, cell-type specific silicification depends on the symplastic pathway of Si delivered by Lsi6 rather than apoplastic pathway. ${ }^{27)}$

\section{Transporter for inter-vascular transfer of Si}

In gramineous plants, minerals taken up by the roots are not directly transported to the grains but are redirected at plant nodes. ${ }^{30-32)}$ Therefore, the distribution of minerals at the nodes is considered to be a key step in the selective control of mineral accumulation in the panicles. At node I beneath the panicles of rice, there are enlarged large and small vascular bundles and diffuse vascular bundles (Fig. 7). Large and small vascular bundles come from the lower nodes and connect to the flag leaf and are markedly enlarged at the node. Diffuse vascular bundles are parallel to and surround the enlarged large vascular bundles. They also are assembled in the upper internode (peduncle) to form regular bundles, which connect toward the panicle tissues. ${ }^{30-32)}$ Therefore, inter-vascular transfer of min- 

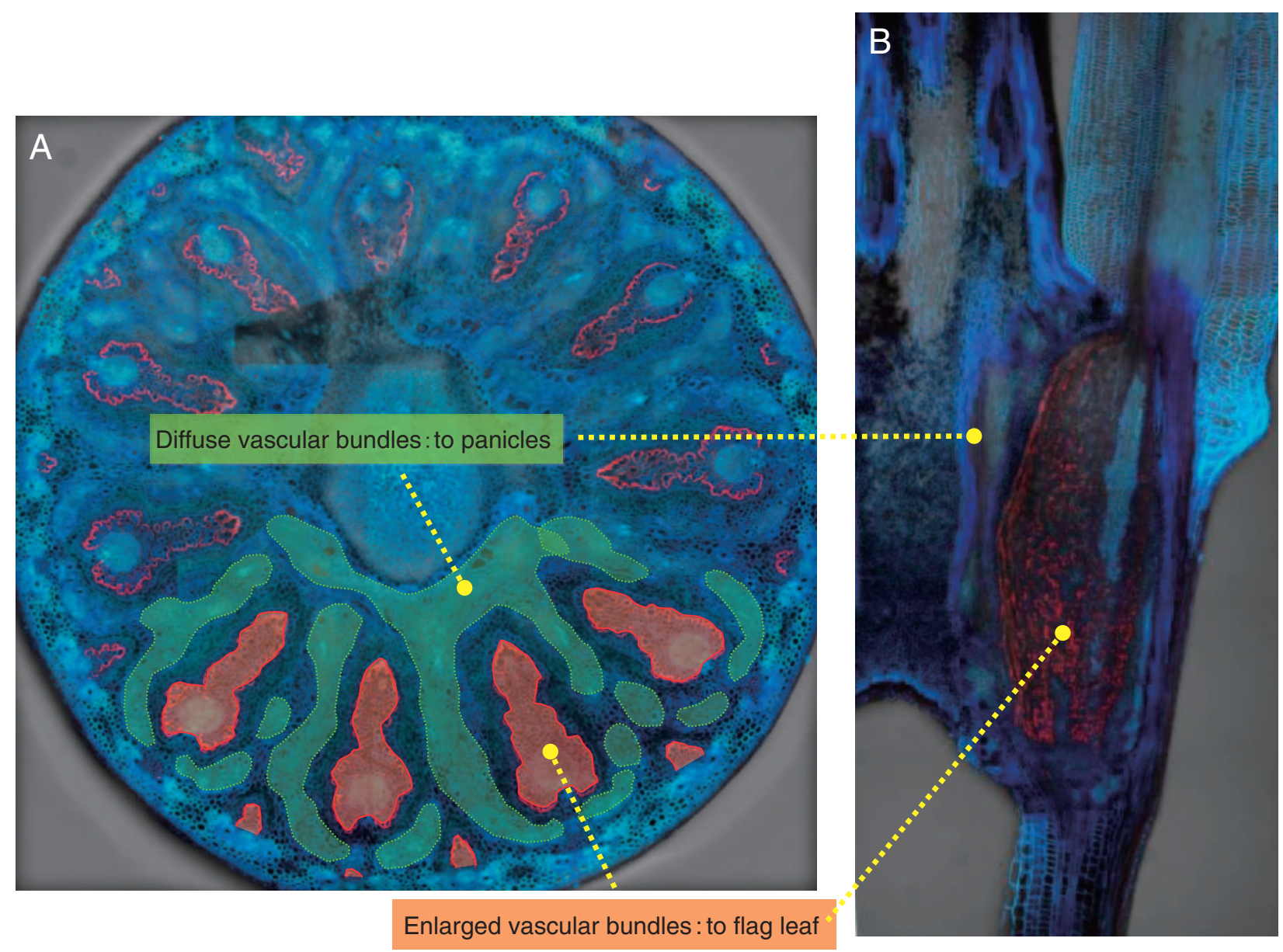

Fig. 7. Localization of Lsi6 at the node I in rice. A, Cross section showing localization of Lsi6 (red) at the transfer cells surrounding enlarged vascular bundles. Enlarged vascular bundles (orange) and diffuse vascular bundles (green) are indicated. B, Longitudinal section of rice node I. Modified from Yamaji and Ma (2009).33)

erals from the enlarged vascular bundles to diffuse vascular bundles is required to deliver minerals taken up by the roots to developing seeds.

Recently, Lsi6 is found to be involved in the inter-vascular transfer of $\mathrm{Si}$ in rice. ${ }^{33)}$ At the reproductive stage, Lsi6 is highly expressed at the node I below the panicles. Lsi6 is mainly localized at the xylem transfer cells with polarity facing toward the xylem vessel (Fig. 7). These cells are located at the outer boundary region of the enlarged vascular bundles and characterized by large surface area due to cell wall ingrowth. Knockout of lsi6 decreased $\mathrm{Si}$ accumulation in the panicles but increased $\mathrm{Si}$ accumulation in the flag leaf. ${ }^{33)}$ These findings suggest that Lsi6 is required for transfer of $\mathrm{Si}$ from the large vascular bundles coming from the roots to the diffuse vascular bundles connected to the panicles.

\section{Concluding remark}

Three different transporters involved in uptake, xylem unloading and inter-vascular transfer of $\mathrm{Si}$ have been identified as described above. However, for transport of Si from soil to the panicles, other transporters are also required. For examples, transporters for xylem loading is necessary, but remain unknown. At the node, after unloading $\mathrm{Si}$ from enlarged vascular bundle by Lsi6, some transporters are required to re-load Si into the diffuse vascular. However, these transporters are unidentified.

Si taken up is finally deposited as silica in specific cells. For example, in rice leaves, silica is deposited in dumbbell-like vascular bundles cells, bulliform motor cells. In rice grain, silica is mostly deposited in the husk. Therefore, there must be other transporters for such cell-specific deposition 
of Si. There transporters remains to be identified in future.

Identification of more $\mathrm{Si}$ transporters from different plant species is also important. Si accumulation differs greatly with plant species. Comparison of the expression patterns and localization of $\mathrm{Si}$ transporters from different plant species may provide new insight into differential species-dependent Si accumulation.

\section{Acknowledgements}

Most work presented in this paper was supported by a Grant-in-Aid for Scientific Research on Innovative Areas from the Ministry of Education, Culture, Sports, Science and Technology of Japan (No. 22119002 to J.F.M.) and a grant from the Ministry of Agriculture, Forestry and Fisheries of Japan, Genomics for Agricultural Innovation IPG0006 (to J.F.M.). We thank Kozo Iwasaki for providing picture of pumpkin.

\section{References}

1) Sommer, M., Kaczorek, D., Kuzyakov, Y. and Breuer, J. (2006) Silicon pools and fluxes in soils and landscapes - a review. J. Plant Nutr. Soil Sci. 169, 310-329.

2) Epstein, E. (1999) Silicon. Annu. Rev. Plant Physiol. Plant Mol. Biol. 50, 641-664.

3) Ma, J.F. and Takahashi, E. (2002) Soil, Fertilizer, and Plant Silicon Research in Japan. Elsevier, Amsterdam.

4) Hodson, M.J., White, P.J., Mead, A. and Broadley, M.R. (2005) Phylogenetic variation in the silicon composition of plants. Ann. Bot. 96, 1027-1046.

5) Arnon, D.I. and Stout, P.R. (1939) The essentiality of certain elements in minute quantity for plants with special reference to copper. Plant Physiol. 14, 371-375.

6) Datnoff, L.E. and Rodrigues, F.A. (2005) The role of silicon in suppressing rice diseases. APSnet Features. http://www.apsnet.org/publications/ apsnetfeatures/Pages/SiliconInRiceDiseases.aspx

7) Fauteux, F., Remus-Borel, W., Menzies, J.G. and Belanger, R.R. (2005) Silicon and plant disease resistance against pathogenic fungi. FEMS Microbiol. Lett. 249, 1-6.

8) Savant, N.K., Snyder, G.H. and Datnoff, L.E. (1997) Silicon management and sustainable rice production. Adv. Agron. 58, 151-199.

9) Cotterill, J.V., Watkins, R.W., Brennon, C.B. and Cowan, D.P. (2007) Boosting silica levels in wheat leaves reduces grazing by rabbits. Pest Manag. Sci. 63, 247-253.

10) Ma, J.F. and Yamaji, N. (2006) Silicon uptake and accumulation in higher plants. Trends Plant Sci. 11, 392-397.

11) Ma, J.F. (2004) Role of silicon in enhancing the resistance of plants to biotic and abiotic stresses. Soil Sci. Plant Nutr. 50, 11-18.

12) Richmond, K.E. and Sussman, M. (2003) Got silicon? The non-essential beneficial plant nutrient. Curr. Opin. Plant Biol. 6, 268-272.

13) Iwasaki, K. and Matsumura, A. (1999) Effect of silicon on alleviation of manganese toxicity in pumpkin (Cucurbita moschata Duch cv. Shintosa). Soil Sci. Plant Nutr. 45, 909-920.

14) Ma, J.F., Nishimura, K. and Takahashi, E. (1989) Effect of silicon on the growth of rice plant at different growth stages. Soil Sci. Plant Nutr. 35, $347-356$.

15) Tamai, K. and Ma, J.F. (2008) Reexamination of silicon effects on rice growth and production under field conditions using a low silicon mutant. Plant Soil 307, 21-27.

16) Ma, J.F., Tamai, K., Ichii, M. and Wu, G.F. (2002) A rice mutant defective in $\mathrm{Si}$ uptake. Plant Physiol. 130, 2111-2117.

17) Ma, J.F., Tamai, K., Yamaji, N., Mitani, N., Konishi, S., Katsuhara, M., Ishiguro, M., Murata, Y. and Yano, M. (2006) A silicon transporter in rice. Nature 440, 688-691.

18) Yamaji, N. and Ma, J.F. (2011) Further characterization of a rice Si efflux transporter, Lsi2. Soil Sci. Plant Nutr. 57, 259-264.

19) Yamaji, N. and Ma, J.F. (2007) Spatial distribution and temporal variation of the rice silicon transporter Lsi1. Plant Physiol. 143, 1306-1313.

20) Chiba, Y., Mitani, N., Yamaji, N. and Ma, J.F. (2009) HvLsi1 is a silicon influx transporter in barley. Plant J. 57, 810-818.

21) Mitani, N., Yamaji, N. and Ma, J.F. (2009) Identification of maize silicon influx transporters. Plant Cell Physiol. 50, 5-12.

22) Mitani, N., Yamaji, N., Ago, Y., Iwasaki, K. and Ma, J.F. (2011) Isolation and functional characterization of an influx silicon transporter in two pumpkin cultivars contrasting in silicon accumulation. Plant J. 66, 231-240.

23) Ma, J.F., Yamaji, N., Mitani, N., Tamai, K., Konishi, S., Fujiwara, T., Katsuhara, M. and Yano, M. (2007) An efflux transporter of silicon in rice. Nature 448, 209-212.

24) Mitani, N., Chiba, Y., Yamaji, N. and Ma, J.F. (2009) Identification and characterization of maize and barley Lsi2-like silicon efflux transporters reveals a distinct silicon uptake system from that in rice. Plant Cell 21, 2133-2142.

25) Casey, W.H., Kinrade, S.D., Knight, C.T.G., Rains, D.W. and Epstein, E. (2003) Aqueous silicate complexes in wheat, Triticum aestivum L. Plant Cell Environ. 27, 51-54.

26) Mitani, N., Ma, J.F. and Iwashita, T. (2005) Identification of the silicon form in xylem sap of rice (Oryza sativa L.). Plant Cell Physiol. 46, 279283.

27) Yamaji, N., Mitani, N. and Ma, J.F. (2008) A transporter regulating silicon distribution in rice shoots. Plant Cell 20, 1381-1389.

28) Prychid, C.J., Rudall, P.J. and Gregory, M. (2004) 
Systematics and biology of silica bodies in monocotyledons. Bot. Rev. 69, 377-440.

29) Taiz, L. and Zeiger, E. (2006) Plant Physiology. Fourth edition. Sinauer, Sunderland, MA.

30) Kawahara, H., Chonan, N. and Matsuda, T. (1974) Studies on morphogenesis in rice plants 7 . The morphology of vascular bundles in the vegetative nodes of the culm. Jpn. J. Crop. Sci. 43, 389-401.

31) Chonan, N., Kawahara, H. and Matsuda, T. (1985) Ultrastructure of elliptical and diffuse bundles in the vegetative nodes of rice. Jpn. J. Crop. Sci. 54, 393-402.

32) Hoshikawa, K. (1989) The Growing Rice Plant. Nobunkyo, Tokyo.

33) Yamaji, N. and Ma, J.F. (2009) Silicon transporter Lsi6 at the node is responsible for inter-vascular transfer of silicon in rice. Plant Cell 21, 2878-2883.

(Received Mar. 1, 2011; accepted May 6, 2011)

\section{Profile}

Jian Feng Ma was born in 1963. He earned his bachelor's degree in Soil Science and Agrochemistry at Nanjing Agricultural University in 1984 and his master degree and Ph.D. in Agricultural Sciences from Kyoto University in 1998 and 1991. He started his research career with studies on the beneficial effects of silicon in rice and performed pioneering work on alleviative effects of silicon on rice growth and productivity under abiotic stress. He was employed as a postdoctoral fellow and researcher in Suntory Institute for Bioorganic Research during 1991-1995. He worked on iron acquisition in gramineous plants and demonstrated the biosynthetic pathway of various phytosiderophores in different plant species and a specific uptake system for phytosiderophore-ferric complex. In 1995, he was employed as an assistant professor in Research Institute for

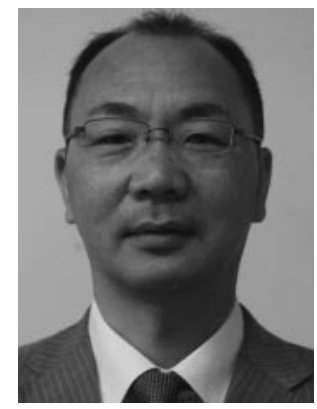
Bioresources of Okayama University and started to work on aluminum tolerance in plants. He found for the first time that high aluminum tolerance of buckwheat is achieved by both external and internal detoxification with oxalate. He moved to Faculty of Agriculture of Kagawa University as an associate professor in 1999 and expanded his work to cadmium uptake and accumulation in hyperaccumulating plants. In 2005, he moved to Research Institute for Bioresources of Okayama University as a full professor and leads a group of Plant Stress Physiology. His group has identified a number of important transporters involved in the uptake, distribution, and accumulation of different minerals including silicon, cadmium, aluminum, arsenic, iron, etc. He is currently a full professor and unit leader of Soil Stress Unit, Institute of Plant Science and Resources, Okayama University. He was awarded the JSPS Prize in 2006, Japan Academy Medal in 2006, and Awards of Japanese Society of Soil Science and Plant Nutrition in 2007. 